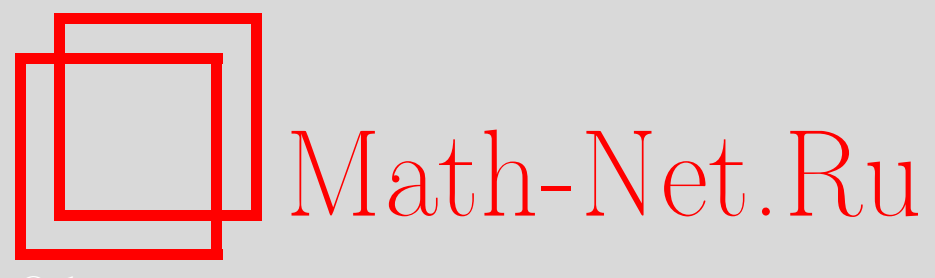

Г. А. Майлыбаева, Границы вырожденности протоколов доступа к данным без раскрытия запроса, Дискрет. матем., 2006, том 18, выпуск 2, 98-110

DOI: https://doi.org/10.4213/dm49

Использование Общероссийского математического портала Math-Net.Ru подразумевает, что вы прочитали и согласны с пользовательским соглашением http://www . mathnet.ru/rus/agreement

Параметры загрузки:

IP : 52.205 .19 .152

26 апреля 2023 г., 13:30:06

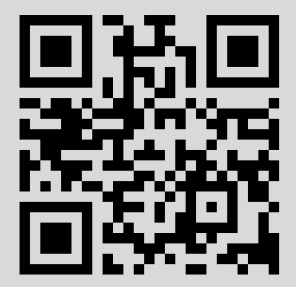




\title{
Границы вырожденности протоколов доступа к данным без раскрытия запроса
}

\author{
() 2006 г. Г. А. Майлыбаева
}

\begin{abstract}
Протоколы извлечения информации без раскрытия запроса позволяют пользователю получить желаемый бит информации из базы данных, копия которой хранится на нескольких несообщающихся серверах, таким образом, что администраторы базы данных ничего не узнают о номере бита, который запрашивал пользователь. Протокол считается вырожденным, если пользователь всегда в ответ получает всю базу данных. В работе найдена граница значений параметров протокола, при которых можно избежать вырожденности.
\end{abstract}

\section{1. Введение}

Рассмотрим протокол с $k+1$ участником: пользователем и $k$ несообщающимися серверами $(k \geqslant 1)$, причем каждый из серверов хранит один и тот же булев вектор $x=\left(x_{0}, \ldots, x_{n-1}\right)$ длины $n-$ базу данных. Пользователь желает узнать значение $i$-го бита $x_{i}$ этого вектора так, чтобы номер бита $i$ не стал известен ни одному из серверов. Протокол который позволяет это делать называется протоколом доступа к данным без раскрытия запроса и состоит из следующих шагов.

(1) Пользователь имеет номер бита $i$ и вырабатывает случайное число $r$. По числам $i$ и $r$ пользователь вычисляет с помощью специальной функщии, называемой функцией запросов, $k$ чисел $q^{j}$ и посылает $j$-му серверу запрос $q^{j}, j=1,2, \ldots, k$.

(2) Каждый из $k$ серверов по полученному запросу $q^{j}$ и базе данных $x$ с помощью спещиальной функщии ответов вычисляет вектор $a^{j}$ и посылает его пользователю.

(3) Пользователь по числам $i, r$ и $k$ ответам серверов $a^{j}$ вычисляет с помощью реконструирующей функщии нужный бит $x_{i}$.

Первое требование к протоколу таково, что ни один из серверов по своему запросу $q^{j}$ не может понять, с помощью какого бита $i$ этот запрос был порожден. Это требование называется требованием защищенности. Второе требование к протоколу, называемое требованием корректности, заключается в том, что пользователь по ответам серверов правильно восстанавливает бит $x_{i}$. Предполагается, что всем участникам протокола, и пользователю, и серверам, известны функции запросов, ответов и реконструирующая. Но серверам не известно случайное число $r$ и, разумеется, не известен номер бита $i$. 
Понятие такого протокола впервые было введено в [1] под названием Private Information Retrieval, поэтому мы в дальнейшем будем называть такие протоколы PIR-протоколами.

Суммарная длина в битах запросов к серверам и ответов серверов называется коммуникационной сложностью PIR-протокола, то есть коммуникационная сложность - это суммарное число бит, которыми обмениваются пользователи и серверы во время протокола.

Существует простейший PIR-протокол, при котором на первом шаге пользователь ничего не делает. На втором шаге каждый из серверов посылает пользователю какуюто свою часть базы данных так, что в сумме пользователь получает всю базу данных. На третьем шаге пользователь выбирает нужный бит базы данных. Коммуникационная сложность этого протокола равна длине базы данных $n$.

PIR-протокол, у которого коммуникационная сложность больше либо равна длине базы данных, будем считать вырожденным.

B работе найдены границы вырожденности PIR-протокола по основным его параметрам. Так показано, что если число серверов $k=1$, то нельзя построить невырожденный PIR-протокол, но уже при $k=2$ существует невырожденный PIR-протокол. Показано, что если $m$ - мощность множества значений функщии запросов PIR-протокола, то при $m \leqslant 1$ не существует, а при $m \geqslant 2$ существует невырожденный PIR-протокол. Показано, что если $s$ - мощность множества значений датчика случайных чисел, используемого в PIR-протоколе, то при $s \leqslant 1$ не существует, а при $s \geqslant 2$ существует невырожденный PIR-протокол. Показано, что если в ответах серверов содержатся только открытые биты базы данных, то есть, если каждый бит ответов существенно зависит только от одного бита базы данных, то не существует невырожденного PIR-протокола, и наоборот, если хотя бы один сервер имеет функцию запросов такую, что биты его ответа существенно зависят хотя бы от двух битов базы данных, то невырожденный PIR-протокол существует.

По поводу реконструирующей функции показано, что если она существенно зависит только от одного бита из ответов серверов, то не существует невырожденного PIR-протокола, но если реконструирующая функция существенно зависит хотя бы от двух битов из ответов серверов, невырожденный PIR-протокол существует.

Автор выражает благодарность Э. Э. Гасанову за постановку задачи.

\section{2. Основные понятия}

Приведем строго формальное определение PIR-протокола, введенное ранее в [2].

Для любого натурального $n$, пусть $E_{n}=\{0,1, \ldots, n-1\}$.

Пусть $k, n, s, m, p^{0}, p^{1}, \ldots, p^{k-1}$ - натуральные числа, $p=p^{0}+p^{1}+\ldots+p^{k-1}$. Пусть на множестве $B=\left\{(i, r), i \in E_{n}, r \in E_{s}\right\}$ задано вероятностное пространство $\left\langle B, 2^{B}, \mathbf{P}\right\rangle$, где $\mathbf{P}\left\{i \in E_{n}, r \in E_{s}\right\}=P(i, r)=1 /(n s)$, для любых $i \in E_{n}, r \in E_{s}$. Тогда $(k, n, s, m, p)$ PIR-протоколом называется набор из $k+2$ функций $I=\left\langle Q, A^{0}, A^{1}, \ldots, A^{k-1}, R\right\rangle$, где $Q, A^{0}, A^{1}, \ldots, A^{k-1}, R$ - некоторые отображения,

$$
\begin{aligned}
& Q: E_{k} \times E_{n} \times E_{s} \rightarrow E_{m}, \\
& R: E_{n} \times E_{s} \times\{0,1\}^{p} \rightarrow\{0,1\} \\
& A^{j}: E_{m} \times\{0,1\}^{n} \rightarrow\{0,1\}^{p^{j}}, \quad j \in E_{k},
\end{aligned}
$$

такие, что выполнены следующие условия 
условие корректности: для любых $i \in E_{n}, r \in E_{s}$

$$
R\left(i, r, A^{0}(Q(0, i, r), x), \ldots, A^{k-1}(Q(k-1, i, r), x)\right)=x_{i} ;
$$

условие защищенности: для любых $q \in E_{m}, t \in E_{k}, i, j \in E_{n}$

$$
\mathbf{P}(Q(t, i, r)=q)=\mathbf{P}(Q(t, j, r)=q) .
$$

Здесь и далее $k$ - число серверов, $n$ - длина базы данных $x=\left(x_{0}, x_{1}, \ldots, x_{n-1}\right), s-$ параметр датчика случайных чисел, точнее, датчик случайных чисел дает равновероятно числа из множества $E_{s}, m$ - характеристика функции запросов $Q$, точнее, функция запросов $Q$ принимает значения из $E_{m}, p^{j}$ - число бит в ответе $j$-го сервера, $A^{j}-$ функция ответа $j$-го сервера, $j \in E_{k}, R$ - реконструирующая функщия.

Содержательно протокол $I=\left\langle Q, A^{0}, \ldots, A^{k-1}, R\right\rangle$ состоит из следующих шагов.

- Пользователь $U$, имея запрос $i$, вырабатывает случайное число $r \in E_{s}$, для каждого $j \in E_{k}$ вычисляет $q^{j}=Q(j, i, r)$ и посылает $j$-му серверу $S_{j}$ число $q^{j}$.

- Каждый сервер $S_{j}, j \in E_{k}$, вычисляет $a^{j}=\left(a_{0}^{j}, \ldots, a_{p^{j}-1}^{j}\right)=A^{j}\left(q^{j}, x\right)$ и посылает вектор $a^{j}$ пользователю.

- $U$ вычисляет $x_{i}=R\left(i, r, a^{0}, \ldots, a^{k-1}\right)$.

Если $d$ - вещественное число, то через ] $d$ [ обозначим наименьшее целое, не меньшее $d$, а через $[d]$ - наибольшее целое, не большее $d$.

Величина

$$
C(I)=k] \log _{2} m[+p
$$

называется коммуникационной сложностью протокола $I$, она равна числу бит, переданных в процессе протокола.

Условие корректности гарантирует, что пользователь получит нужный бит базы данных, а условие защищенности гарантирует, что ни один из серверов по вектору $q$, который он получил, не сможет понять, какой бит интересует пользователя.

Основной целью исследований в этой области является построение для заданного числа серверов $k$, длины базы данных $n$ и максимального значения датчика случайных чисел $s$ PIR-протокола с минимальной коммуникационной сложностью.

\section{3. Простейший вырожденный PIR-протокол}

Рассмотрим следующий $(k, n, 1,1, n)$ PIR-протокол $I_{b}=\left\langle Q, A^{0}, \ldots, A^{k-1}, R\right\rangle$. Поскольку $s=1$, у него фактически отсутствует датчик случайных чисел. Так как $m=1$, функщия запросов есть тождественная функция, то есть $Q(j, i, 0) \equiv 0$ для любых $j \in E_{k}, i \in E_{n}$, что содержательно означает, что пользователь на первом шаге PIR-протокола ничего не делает. Для $x \in\{0,1\}^{n}$ и $j \in E_{k-1}$ положим

$$
\begin{aligned}
A^{j}(0, x) & =A^{j}(x)=\left(x_{j] n / k[}, \ldots, x_{(j+1)] n / k[-1}\right), \\
A^{k-1}(0, x) & =A^{k-1}(x)=\left(x_{j] n / k[}, \ldots, x_{n-1}\right) .
\end{aligned}
$$


Положим

$$
R\left(i, A^{0}(x), \ldots, A^{k-1}(x)\right)=R(i, x)=x_{i} .
$$

Содержательно это означает, что каждый сервер выдает пользователю свою часть базы данных, а пользователь, собрав всю базу данных, извлекает нужный бит. Понятно, что сложность этого протокола равна

$$
\left.C\left(I_{b}\right)=k\right] \log _{2} m[+n=n .
$$

Отметим также, что каждый бит ответов серверов есть открытый бит базы данных, то есть каждый бит ответа существенно зависит только от одного бита базы данных. Кроме того, при любом фиксированном $i$ реконструирующая функщия существенно зависит только от одного бита из ответов серверов.

\section{4. Простейший невырожденный PIR-протокол}

Опишем невырожденный PIR-протокол $I_{g}=\left\langle Q, A^{0}, A^{1}, R\right\rangle$ для двух серверов с датчиком случайных чисел, принимающем два значения, $s=2$, и $m=2$.

Введем обозначения

$$
l^{\prime}=[n / 4], \quad l^{\prime \prime}=n \quad(\bmod 4)=n-4 l^{\prime}
$$

Протокол состоит из трех шагов.

Шаг 1. По номеру бита $i$ и случайному числу $r \in E_{2}$ пользователь вычисляет $q^{j}$, $j \in E_{2}$, по правилу $q^{0}=Q(0, i, r)=r$,

$$
q^{1}=Q(1, i, r)= \begin{cases}r, & \text { если } i<2 l^{\prime}, \\ (1+r) \quad(\bmod 2), & \text { если } i \geqslant 2 l^{\prime},\end{cases}
$$

и посылает $j$-му серверу запрос $q^{j}$.

Шаг 2. Каждый бит ответа первого сервера на любой запрос пользователя представляет собой сумму по модулю 2 ровно двух бит базы данных.

Второй сервер на любой запрос пользователя всегда посылает в качестве ответа некоторую проекцию базы данных, а именно, на первый запрос он посылает одну половину бит базы данных, на второй запрос - другую половину бит.

Далее будем полагать, что $M(n, m)$ - множество $n \times m$ матриц с элементами из $\{0,1\}$. Если

$$
B=\left(\begin{array}{ccc}
a_{0,0} & \ldots & a_{0, m-1} \\
& \ldots & \\
a_{n-1,0} & \ldots & a_{n-1, m-1}
\end{array}\right) \in M(n, m),
$$

то положим

$$
B^{T}=\left(\begin{array}{ccc}
a_{0,0} & \ldots & a_{n-1,0} \\
& \ldots & \\
a_{0, m-1} & \ldots & a_{n-1, m-1}
\end{array}\right) \in M(m, n) .
$$


Для вычисления ответа сервер $S_{j}, j \in E_{2}$, использует матрицы $B_{0}^{j}, B_{1}^{j}$, которые будут описаны ниже, причем $B_{0}^{0}, B_{1}^{0} \in M\left(l^{\prime}+l^{\prime \prime}, n\right)$, а $B_{0}^{1}, B_{1}^{1} \in M\left(2 l^{\prime}, n\right)$. Предполагается, что пользователь знает вид этих матриц.

Сервер $S_{j}$ вычисляет ответ $a^{j}$, используя формулу $a^{j}=A^{j}\left(q^{j}, x\right)=\left(B_{q^{j}}^{j} x^{T}\right)^{T}$, и посылает результат пользователю.

Пусть $E$ - единичная матрица из $M\left(l^{\prime}, l^{\prime}\right), O$ - нулевая матрица из $M\left(l^{\prime}, l^{\prime}\right)$, то есть

$$
E=\left(\begin{array}{cccc}
1 & 0 & \ldots & 0 \\
0 & 1 & \ldots & 0 \\
& & \ldots & \\
0 & 0 & \ldots & 1
\end{array}\right), \quad O=\left(\begin{array}{cccc}
0 & 0 & \ldots & 0 \\
0 & 0 & \ldots & 0 \\
& & \ldots & \\
0 & 0 & \ldots & 0
\end{array}\right)
$$

Если $l^{\prime \prime} \neq 0$, то пусть $E^{\prime}$ - единичная матрища из $M\left(l^{\prime \prime}, l^{\prime \prime}\right), O^{\prime}-$ нулевая матрица из $M\left(l^{\prime}, l^{\prime \prime}\right), O^{\prime \prime}$ - нулевая матрица из $M\left(l^{\prime \prime}, l^{\prime}\right)$.

Тогда матрицы, используемые сервером $S_{0}$, имеют вид

$$
B_{0}^{0}=\left(\begin{array}{ccccc}
E & O & O & E & O^{\prime} \\
O^{\prime \prime} & O^{\prime \prime} & O^{\prime \prime} & O^{\prime \prime} & E^{\prime}
\end{array}\right), \quad B_{1}^{0}=\left(\begin{array}{ccccc}
O & E & E & O & O^{\prime} \\
O^{\prime \prime} & O^{\prime \prime} & O^{\prime \prime} & O^{\prime \prime} & E^{\prime}
\end{array}\right),
$$

матрищы, используемые сервером $S_{1}$, имеют вид

$$
B_{0}^{1}=\left(\begin{array}{lllll}
O & E & O & O & O^{\prime} \\
O & O & O & E & O^{\prime}
\end{array}\right), \quad B_{1}^{1}=\left(\begin{array}{ccccc}
E & O & O & O & O^{\prime} \\
O & O & E & O & O^{\prime}
\end{array}\right)
$$

Шаг 3. Пользователь действует по следующей схеме.

1. Если $q^{0}=r=0$, то

(a) если $i<2 l^{\prime}$, то $q^{1}=0$ и

(i) если $i<l^{\prime}$, то $x_{i}$ получается как сумма двух бит из ответов обоих серверов, а именно, $x_{i}=a_{i}^{0}+a_{l^{\prime}+i}^{1}$,

(ii) если же $l^{\prime} \leqslant i<2 l^{\prime}$, то $x_{i}$ извлекается из ответа второго сервера, а именно, $x_{i}=a_{i-l^{\prime}}^{1}$,

(b) если $2 l^{\prime} \leqslant i<4 l^{\prime}$, то $q^{1}=1$ и

(i) если $2 l^{\prime} \leqslant i<3 l^{\prime}$, то $x_{i}$ извлекается из ответа второго сервера, а именно, $x_{i}=a_{i-l^{\prime}}^{1}$

(ii) если же $3 l^{\prime} \leqslant i<4 l^{\prime}$, то $x_{i}$ получается как сумма двух бит из ответов обоих серверов, а именно, $x_{i}=a_{i-3 l^{\prime}}^{0}+a_{i-3 l^{\prime}}^{1}$,

(c) если $i \geqslant 4 l^{\prime}, x_{i}$ извлекается из ответа первого сервера, а именно, $x_{i}=a_{i-3 l^{\prime}}^{0}$,

2. Если $q^{0}=r=1$, то

(a) если $i<2 l^{\prime}$, то $q^{1}=1$ и

(i) если $i<l^{\prime}$, то $x_{i}$ извлекается из ответа второго сервера, а именно, $x_{i}=a_{i}^{1}$,

(ii) если же $l^{\prime} \leqslant i<2 l^{\prime}$, то $x_{i}$ получается как сумма двух бит из ответов обоих серверов, а именно, $x_{i}=a_{i-l^{\prime}}^{0}+a_{i}^{1}$,

(b) если $2 l^{\prime} \leqslant i<4 l^{\prime}$, то $q^{1}=0$ и 
(i) если $2 l^{\prime} \leqslant i<3 l^{\prime}$, то $x_{i}$ получается как сумма двух бит из ответов обоих серверов, а именно, $x_{i}=a_{i-2 l^{\prime}}^{0}+a_{i-2 l^{\prime}}^{1}$

(ii) если же $3 l^{\prime} \leqslant i<4 l^{\prime}$, то $x_{i}$ извлекается из ответа второго сервера, а именно, $x_{i}=a_{i-2 l^{\prime}}^{1}$

(c) если $i \geqslant 4 l^{\prime}, x_{i}$ извлекается из ответа первого сервера, а именно, $x_{i}=a_{i-3 l^{\prime}}^{0}$,

На языке матриц реконструирующую функцию можно описать следующим образом.

Используя матрицы $B_{q^{0}}^{0}, B_{q^{1}}^{1}$ и ответы серверов $a^{0}, a^{1}$, пользователь строит систему из $2 l^{\prime}$ уравнений с $2 l^{\prime}$ неизвестными - битами базы данных. Таким образом, пользователь вычисляет значение бита $x_{i}$ как линейную комбинацию бит ответов серверов. А именно, для запросов $q^{0}, q^{1}$ расширенная матрица $R_{q^{0}, q^{1}}^{\prime}$ системы линейных уравнений для переменных $x_{0}, \ldots, x_{2} l^{\prime-1}$ имеет вид

$$
R_{q^{0}, q^{1}}^{\prime}=\left(\begin{array}{cc}
E & O \\
O & E
\end{array} \mid C_{q^{0}, q^{1}}^{\prime}\right),
$$

где $\left(q^{0}, q^{1}\right) \in\{(0,0),(1,1)\}$ и $C_{q^{0}, q^{1}}^{\prime} \in M(n / 2,1)$,

$$
C_{0,0}^{\prime}=\left(\begin{array}{c}
a_{0}^{0}+a_{l^{\prime}}^{1} \\
\cdots \\
a_{l^{\prime}-1}^{0}+a_{2 l^{\prime}-1}^{1} \\
a_{0}^{1} \\
\cdots \\
a_{l^{\prime}-1}^{1}
\end{array}\right), \quad C_{1,1}^{\prime}=\left(\begin{array}{c}
a_{0}^{1} \\
\cdots \\
a_{l^{\prime}-1}^{1} \\
a_{0}^{0}+a_{l^{\prime}}^{1} \\
\cdots \\
a_{l^{\prime}-1}^{0}+a_{2 l^{\prime}-1}^{1}
\end{array}\right) .
$$

Расширенная матрица $R_{q^{0}, q^{1}}^{\prime \prime}$ системы уравнений для $x_{2 l^{\prime}}, \ldots, x_{4 l^{\prime}-1}$ имеет вид

$$
R_{q^{0}, q^{1}}^{\prime \prime}=\left(\begin{array}{cc}
E & O \\
O & E
\end{array} \mid C_{q^{0}, q^{1}}^{\prime \prime}\right)
$$

где $\left(q^{0}, q^{1}\right) \in\{(0,1),(1,0)\}, C_{q^{0}, q^{1}}^{\prime \prime} \in M(n / 2,1)$,

$$
C_{0,1}^{\prime \prime}=\left(\begin{array}{c}
a_{l^{\prime}}^{1} \\
\cdots \\
a_{2 l^{\prime}-1}^{1} \\
a_{0}^{0}+a_{0}^{1} \\
\cdots \\
a_{l^{\prime}-1}^{0}+a_{l^{\prime}-1}^{1}
\end{array}\right), \quad C_{1,0}^{\prime \prime}=\left(\begin{array}{c}
a_{0}^{0}+a_{0}^{1} \\
\cdots \\
a_{l^{\prime}-1}^{0}+a_{l^{\prime}-1}^{1} \\
a_{l^{\prime}}^{1} \\
\cdots \\
a_{2 l^{\prime}-1}^{1}
\end{array}\right) .
$$

Расширенная матрица $R^{\prime \prime \prime}$ системы уравнений для $x_{4} l^{\prime}, \ldots, x_{n}$ имеет вид

$$
R^{\prime \prime \prime}=\left(E^{\prime} \mid C^{\prime \prime \prime}\right)
$$

где

$$
C^{\prime \prime \prime}=\left(\begin{array}{c}
a_{2 l^{\prime}}^{0} \\
\cdots \\
a_{2 l^{\prime}+l^{\prime \prime}-1}^{0}
\end{array}\right) .
$$

Легко видеть, что сложность этого протокола равна

$$
C\left(I_{g}\right)=3 l^{\prime}+l^{\prime \prime}+2<n
$$


при $n \geqslant 12$.

Отметим, что один из серверов посылает в ответ только открытые биты базы данных, а в ответе другого каждый бит есть сумма не более двух бит базы данных. При этом при любых фиксированных $r \in E_{s}, i \in E_{n}$ реконструирующая функция зависит от не более чем двух бит из ответов серверов.

\section{5. Граница вырожденности по числу серверов}

Докажем одно простое свойство PIR-протоколов.

Свойство 1. Для любого $(k, n, s, m, p)$ PIR-протокола справедливо равенство $s=\operatorname{lm}$, где $l$ - некоторое натуральное число, и для любых $j \in E_{k}, i \in E_{n}, q \in E_{m}$ справедливо равенство $\left|\left\{r \in E_{s}: Q(j, i, r)=q\right\}\right|=s / m=l$.

Доказательство. В силу защищенности PIR-протокола для любых $j \in E_{k}, i \in E_{n}$ и $q, q^{\prime} \in E_{m}$ справедливо равенство

$$
\mathbf{P}(Q(j, i, r)=q)=\mathbf{P}\left(Q(j, i, r)=q^{\prime}\right) .
$$

Отсюда, учитывая условие равномерности вероятностной меры, заключаем, что

$$
\left|\left\{r \in E_{s}: Q(j, i, r)=q\right\}\right|=\left|\left\{r \in E_{s}: Q(j, i, r)=q^{\prime}\right\}\right| .
$$

Отсюда сразу следует, что $s$ делится нацело на $m$ и $\left|\left\{r \in E_{s}: Q(j, i, r)=q\right\}\right|=s / m$.

Предложение 1. Если число серверов равно $k=1$, то не существует невырожденный

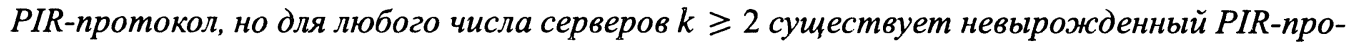
токол.

Доказательство. Сначала покажем, что для любого $(1, n, s, m, p)$ PIR-протокола

$$
I=\left\langle Q, A^{0}, R\right\rangle
$$

справедливо неравенство

$$
C(I) \geqslant n
$$

Этот результат упоминался в [1]. В данной модели он получает простое доказательство.

Покажем, что длина $p$ вектора $A^{0}(q, x)$ не меньше чем $n$. Предположим обратное. Зафиксируем некоторый запрос $q^{0} \in E_{m}$. Тогда $\left|\left\{A^{0}\left(q^{0, x}\right): x \in\{0,1\}^{n}\right\}\right|<2^{n}$. Тогда существуют $x^{\prime}, x^{\prime \prime} \in\{0,1\}^{n}, x^{\prime} \neq x^{\prime \prime}$, такие, что $A^{0}\left(q^{0}, x^{\prime}\right)=A^{0}\left(q^{0}, x^{\prime \prime}\right)$. Поскольку $x^{\prime} \neq x^{\prime \prime}$, существует такой номер $l \in E_{n}$, что $x_{l}^{\prime} \neq x_{l}^{\prime \prime}$. Согласно свойству 1 , сушествует такое число $r^{0} \in E_{s}$, что $Q\left(0, l, r^{0}\right)=q^{0}$. По условию корректности

$$
x_{l}^{\prime}=R\left(l, r^{0}, A^{0}\left(q^{0}, x^{\prime}\right)\right)=R\left(l, r^{0}, A^{0}\left(q^{0}, x^{\prime \prime}\right)\right)=x_{l}^{\prime \prime} .
$$

Это противоречие доказывает, что $p \geqslant n$. Следовательно,

$$
C(I)=m+p \geqslant p \geqslant n,
$$

и значит, любой PIR-протокол с одним сервером вырожденный, а пример протокола $I_{b}$ из раздела 3 показывает, что вырожденный PIR-протокол существует.

Существование невырожденного PIR-протокола для более одного сервера доказывает протокол $I_{g}$ из раздела 4. 


\section{6. Граница вырожденности по длине запроса}

Предложение 2. Если $m$ - мощность множества значений функции запросов, то для $m=1$ не существует, а для $m \geqslant 2$ существует невырожденный PIR-протокол.

Доказательство. Сначала покажем, что для любого $(k, n, s, 1, p)$ PIR-протокола

$$
I=\left\langle Q, A^{0}, \ldots, A^{k-1}, R\right\rangle
$$

справедливо неравенство

$$
C(I) \geqslant n \text {. }
$$

То, что $m=1$, означает, что длина запроса $] \log m[=0$, и значит, на первом шаге пользователь не посылает серверам запрос, и следовательно, функция ответа каждого сервера и реконструирующая функция не зависят от запроса. Рассмотрим вектор $\left(A^{0}(x), \ldots, A^{k-1}(x)\right)$, покажем что длина этого вектора не менее чем $n$. Предположим обратное. Тогда существует две базы данных $x^{\prime}, x^{\prime \prime} \in\{0,1\}^{n}, x^{\prime} \neq x^{\prime \prime}$ такие, что $\left(A^{0}\left(x^{\prime}\right), \ldots, A^{k-1}\left(x^{\prime}\right)\right)=\left(\left(A^{0}\left(x^{\prime \prime}\right), \ldots, A^{k-1}\left(x^{\prime \prime}\right)\right)\right.$. Поскольку $x^{\prime} \neq x^{\prime \prime}$, существует такой номер $l \in E_{n}$, что $x_{l}^{\prime} \neq x_{l}^{\prime \prime}$. По условию корректности получаем, что

$$
x_{l}^{\prime}=R\left(l, 0, A^{0}\left(x^{\prime}\right), \ldots, A^{k-1}\left(x^{\prime}\right)\right)=R\left(l, 0, A^{0}\left(x^{\prime \prime}\right), \ldots, A^{k-1}\left(x^{\prime \prime}\right)\right)=x_{l}^{\prime \prime} .
$$

Это противоречие доказывает неравенство $C(I) \geqslant n$.

Существование невырожденного PIR-протокола для $m \geqslant 2$ доказывает протокол $I_{g}$ из раздела 4.

\section{7. Граница вырожденности по мощности множества значений датчика случайных чисел}

Предложение 3. Если $s$ - мочность множества значений датчика случайных чисел, то для $s=1$ не существует, а для $s \geqslant 2$ существует невырожденный PIR-протокол.

Доказательство. Согласно свойству $1 s \geqslant m$, где $m$ - мощность множества значений функции запросов. Тогда из равенства $s=1$, следует, что $m=1$, и согласно предложению 2 , в этом случае не существует невырожденного протокола.

Существование невырожденного PIR-протокола для $s \geqslant 2$ доказывает протокол $I_{g}$ из раздела 4.

\section{8. Граница вырожденности по длине базы данных}

Для любого натурального $n$ обозначим $S_{n}$ множество всех перестановок из $E_{n}$, другими словами, множество всех взаимно однозначных функций $\pi: E_{n} \rightarrow E_{n}$.

Лемма 1. Если число бит в базе данных равно $n, k$ - число серверов, $m-$ мочность множества значений функции запросов, $p^{j}-$ число бит в ответе $j$-го сервера, то для любого PIR-протокола и любой перестановки $\pi \in S_{k}$

$$
p^{\pi(0)}+m\left(p^{\pi(1)}+\ldots+p^{\pi(k-1)}\right) \geqslant n .
$$


Доказательство. Рассмотрим произвольный $(k, n, s, m, p)$ PIR-протокол

$$
I=\left\langle Q, A^{0}, \ldots, A^{k-1}, R\right\rangle,
$$

где $p=p^{0}+p^{1}+\ldots+p^{k-1}$.

Пусть $x \in\{0,1\}^{n}, q \in E_{m}, \pi \in S_{k}$. Рассмотрим вектор

$$
\begin{aligned}
\mathscr{A}(q, x)= & \left(A^{\pi(0)}(q, x),\right. \\
& \left.A^{\pi(1)}(0, x), \ldots, A^{\pi(1)}(m-1, x), \ldots, A^{\pi(k-1)}(0, x), \ldots, A^{\pi(k-1)}(m-1, x)\right) .
\end{aligned}
$$

Длина вектора $\mathscr{A}(q, x)$ равна $p^{\pi(0)}+m\left(p^{\pi(1)}+\ldots+p^{\pi(k-1)}\right)$.

Пусть отображение $R^{\prime}: E_{n} \times E_{s} \times\{0,1\}^{p^{\pi(0)}+m\left(p^{\pi(1)}+\ldots+p^{\pi(k-1)}\right)} \rightarrow\{0,1\}$ задано следующим образом:

$$
\begin{aligned}
R^{\prime}(i, r, \mathscr{A}(Q(\pi(0), i, r), x))=R\left(i, r, A^{\pi(0)}(Q(\pi(0), i, r), x),\right. & \\
& \left.\ldots, A^{\pi(k-1)}(Q(\pi(k-1), i, r), x)\right)=x_{i} .
\end{aligned}
$$

Предположим, что длина вектора $\mathscr{A}$ меньше чем $n$. Тогда существует число $q \in E_{m}$ и два вектора $x^{\prime}, x^{\prime \prime} \in\{0,1\}^{n}, x^{\prime} \neq x^{\prime \prime}$, такие, что $\mathscr{A}\left(q, x^{\prime}\right)=\mathscr{A}\left(q, x^{\prime \prime}\right)$.

Поскольку $x^{\prime} \neq x^{\prime \prime}$, сушествует $l \in E_{n}$ такое, что $x_{l}^{\prime} \neq x_{l}^{\prime \prime}$. Пусть $r$ таково, что $Q(\pi(0), l, r)=q$. Тогда

$$
x_{l}^{\prime}=R^{\prime}\left(l, r, \mathscr{A}\left(q, x^{\prime}\right)\right)=R^{\prime}\left(l, r, \mathscr{A}\left(q, x^{\prime \prime}\right)\right)=x_{l}^{\prime \prime} .
$$

Это противоречие доказывает, что

$$
p^{\pi(0)}+m\left(p^{\pi(1)}+\ldots+p^{\pi(k-1)}\right) \geqslant n .
$$

Предложение 4. Если длина базы данных $n \leqslant 8$, то не существует невырожденного $P I R$-протокола, а при любом $n \geqslant 12$ существует невырожденный PIR-протокол.

Доказательство. Рассмотрим произвольный $(k, n, s, m, p)$ PIR-протокол

$$
I=\left\langle Q, A^{0}, \ldots, A^{k-1}, R\right\rangle,
$$

где $p=p^{0}+p^{1}+\ldots+p^{k-1}$. Если протокол невырожденный, то сложность этого протокола

$$
C(I)=k] \log _{2} m[+p=k] \log _{2} m\left[+p^{0}+p^{1}+\ldots+p^{k-1}<n .\right.
$$

Без ограничения общности можно считать, что $p^{0} \leqslant p^{1} \leqslant \ldots \leqslant p^{k-1}$. Покажем, что при любых значениях $m$ мощности множества значений функции запросов и числа $k$ серверов при $n \leqslant 8$ не существует невырожденного PIR-протокола.

I. Рассмотрим протоколы, в которых участвуют два сервера, $k=2$.

1. Если $n=8$, то для любого невырожденного PIR-протокола

$$
C(I)=2] \log _{2} m\left[+p^{0}+p^{1}<8 \Longrightarrow p^{0}+p^{1}<8-2\right] \log _{2} m[\text {. }
$$


(a) $m=2$, тогда $p^{0}+p^{1}<6$.

Если $p^{0}=1, p^{1}=4$, то $p^{1}+2 p^{0}=6<8=n$, получаем противоречие с леммой 1.

Если $p^{0}=2, p^{1}=3$, то $p^{1}+2 p^{0}=7<8=n$, получаем противоречие.

(b) $m=3,4$, тогда $p^{0}+p^{1}<4, p^{0}=1, p^{1}=2, p^{1}+m p^{0} \leqslant p^{1}+4 p^{0}=6<$ $8=n$, получаем противоречие.

(c) $m \geqslant 5$, тогда $p^{0}+p^{1}<2$. Тогда один из серверов ничего не посылает в ответ пользователю, получаем протокол с одним сервером, который всегда является вырожденным.

2. Если $n=7$, то

$$
\left.p^{0}+p^{1}<7-2\right] \log _{2} m[.
$$

(a) $m=2$, тогда $p^{0}+p^{1}<5$.

Если $p^{0}=2, p^{1}=2$, то $p^{0}+2 p^{1}=6<7=n$, получаем противоречие.

Если $p^{0}=1, p^{1}=3$, то $p^{1}+2 p^{0}=5<7=n$, получаем противоречие.

(b) $m=3,4$, тогда $p^{0}+p^{1}<3, p^{0}=p^{1}=1, p^{0}+m p^{1} \leqslant p^{0}+4 p^{1}=5<$ $7=n$, получаем противоречие.

(c) $m \geqslant 5$, тогда $p^{0}+p^{1}<1$, получаем противоречие.

3. Если $n=6$, то

$$
\left.p^{0}+p^{1}<6-2\right] \log _{2} m[\text {. }
$$

(a) $m=2$, тогда $p^{0}+p^{1}<4, p^{0}=1, p^{1}=2, p^{1}+2 p^{0}=4<6=n$, получаем противоречие.

(b) $m \geqslant 3$, тогда $p^{0}+p^{1}<2$. Получаем протокол для одного сервера.

4. Если $n=5$, то

$$
\left.p^{0}+p^{1}<5-2\right] \log _{2} m[\text {. }
$$

(a) $m=2$, тогда $p^{0}+p^{1}<3, p^{0}=p^{1}=1, p^{0}+2 p^{1}=3<5=n$, получаем противоречие.

(b) $m \geqslant 3$, тогда $p^{0}+p^{1}<1$, получаем противоречие.

5. Если $n \leqslant 4$, то

$$
\left.p^{0}+p^{1}<n-2\right] \log _{2} m[\leqslant 4-2] \log _{2} m[\leqslant 2
$$

для любого натурального $m$. Это означает, что в протоколе участвует ровно один сервер.

II. Рассмотрим протоколы, для которых $k=3$.

1. Если $n=8$, то для любого невырожденного протокола

$$
\left.p^{0}+p^{1}+p^{2}<8-3\right] \log _{2} m[\text {. }
$$

(a) $m=2$, тогда $p^{0}+p^{1}+p^{2}<5, p^{0}=p^{1}=1, p^{2}=2, p^{2}+2\left(p^{0}+p^{1}\right)=$ $6<8=n$, получаем противоречие.

(b) $m \geqslant 3$, тогда $p^{0}+p^{1}+p^{2}<2$. Получаем протокол для одного сервера. 
2. Если $n=7$, то

$$
\left.p^{0}+p^{1}+p^{2}<7-3\right] \log _{2} m[\text {. }
$$

(a) $m=2$, тогда $p^{0}+p^{1}+p^{2}<4, p^{0}=p^{1}=p^{2}=1, p^{2}+2\left(p^{0}+p^{1}\right)=$ $5<7=n$, получаем противоречие.

(b) $m \geqslant 3$, тогда $p^{0}+p^{1}+p^{2}<1$, получаем противоречие.

3. Если $n \leqslant 6$, то

$$
\left.p^{0}+p^{1}+p^{2}<6-3\right] \log _{2} m[\leqslant 3
$$

для любого $m$. Получаем протокол для двух серверов.

III. Рассмотрим протоколы, для которых $k \geqslant 4$.

Если $n \leqslant 8$, то для любого невырожденного протокола и для любого натурального $m$

$$
\left.p^{0}+p^{1}+p^{2}+p^{3}<n-4\right] \log _{2} m[\leqslant 8-4] \log _{2} m[\leqslant 4 .
$$

Это означает, что хотя бы один сервер ничего не посылает в ответ пользователю, получаем протокол для трех серверов.

Существование невырожденного PIR-протокола при $n \geqslant 12$ доказывает PIR-протокол . $I_{g}$ из раздела 4.

\section{9. Граница вырожденности по функции ответов}

Степенью существенности булевой функции $f\left(x_{1}, \ldots, x_{l}\right)$ назовем число существенных переменных, от которых она зависит, и обозначим ее через $S(f)$.

Степенью существенности булевой вектор-функции

$$
F\left(x_{1}, \ldots, x_{l}\right)=\left(f_{1}\left(x_{1}, \ldots, x_{l}\right), \ldots, f_{t}\left(x_{1}, \ldots, x_{l}\right)\right)
$$

назовем число

$$
S(F)=\max _{1 \leqslant j \leqslant t} S\left(f_{j}\right)
$$

Степенью существенности функции ответов $j$-го сервера $A^{j}: E_{m} \times\{0,1\}^{n} \rightarrow\{0,1\}^{p^{j}}$, $j \in E_{k}$, назовем число

$$
S\left(A^{j}\right)=\max _{q \in E_{m}} S\left(A^{j}(q)\right) .
$$

Здесь под $A^{j}(q)$ понимается булева вектор-функция, получающаяся из $A^{j}$ при фиксации запроса $q$.

Предложение 5. Если для любого сервера его функция ответов имеет степень существенности не более 1, то не существует невырожденного PIR-протокола, а если степень существенности функчии ответов хотя бы одного сервера строго больше 1, то существует невырожденный PIR-протокол. 
Доказательство. Обозначим через $\mathscr{A}_{0}$ множество таких PIR-протоколов, что степень существенности функции ответов каждого сервера не превосходит 1. Это означает, что каждый бит ответа есть некоторый бит базы данных или его отрицание. Так как функии ответа известны, можно считать, что каждый бит ответа есть некоторый бит базы данных, или другими словами, функция ответа каждого сервера на любой запрос есть некоторая проекщия базы данных.

Покажем, что для любого $(k, n, s, m, p)$ PIR-протокола

$$
I=\left\langle Q, A^{0}, \ldots, A^{k-1}, R\right\rangle \in \mathscr{A}_{0}
$$

справедливо неравенство

$$
C(I) \geqslant n \text {. }
$$

Рассмотрим вектор $A^{j}(q, x)$, где $j \in E_{k}$ - номер сервера, $q \in E_{m}$ - запрос, $x \in\{0,1\}^{n}$ - база данных. Итак, каждая компонента $A^{j}(q, x)$ есть бит базы данных, то есть $A^{j}(q, x)=\left(x_{i_{1}}, \ldots, x_{i_{p^{j}}}\right)$. Обозначим через $b_{j, q}=\left\{i_{1}, \ldots, i_{p^{j}}\right\}$ множество номеров бит из $A^{j}(q, x)$.

Введем обозначение

$$
B=\left\{b_{j, q}: j \in E_{k}, q \in E_{m}\right\} .
$$

Покажем, что для каждого $i \in E_{n}$ найдется не менее $m$ множеств $b_{j, q}$ из $B$ таких, что $i \in b_{j, q}$. Предположим противное, то есть предположим, что существует номер $i_{0}$, для которого существует $t, t<m$, множеств $b_{j_{1}, q_{1}}, \ldots, b_{j_{t}, q_{t}}$ из $B$ таких, что $i_{0} \in b_{j_{l}, q_{l}}$, $l=1, \ldots, t$. Тогда существует запрос $q_{0}$ такой, что $q_{0} \notin\left\{q_{1}, \ldots, q_{t}\right\}$, и $i_{0} \notin b_{j, q_{0}}$ для любого $j \in E_{k}$. Следовательно, как только какой-либо сервер получит запрос $q_{0}$, он сразу же будет знать, что $i_{0}$-й бит базы данных не интересует пользователя, что противоречит свойству защищенности протокола. Это противоречие доказывает, что для любого $i \in E_{n}$ найдется не менее $m$ множеств $b_{j, q}$ из $B$ таких, что $i \in b_{j, q}$.

Следовательно,

$$
\sum_{q \in E_{m}} \sum_{j \in E_{k}}\left|a_{j, q}\right|=m \sum_{j \in E_{k}} p^{j} \geqslant m n \text {. }
$$

Откуда следует, что

$$
C(I) \geqslant p^{0}+\ldots+p^{k-1} \geqslant n .
$$

Таким образом первая часть предложение 5 доказана.

Для доказательства второй части предложения 5 достаточно заметить, что у PIR-протокола $I_{g}$ из раздела 4 функция ответа первого сервера имеет степень существенности 2 , а второго сервера - степень существенности 1 .

\section{0. Граница вырожденности по реконструирующей функции}

Степенью существенности реконструирующей функщии $R: E_{n} \times E_{s} \times\{0,1\}^{p} \rightarrow\{0,1\}$ назовем число

$$
S(R)=\max _{i \in E_{n}, r \in E_{s}} S(R(i, r))
$$

Здесь под $R(i, r)$ понимается булева функция, получающаяся из $R$ при фиксации номера $i$ и случайного числа $r$. 
Предложение 6. Если степень существенности реконструирующей функции менее 2, то не существует невырожденного PIR-протокола, а если степень существенности реконструирующей функции более 1, то невырожденный PIR-протокол существует.

Доказательство. Покажем, что для любого $(k, n, s, m, p)$ PIR-протокола

$$
I=\left\langle Q, A^{0}, \ldots, A^{k-1}, R\right\rangle
$$

такого, что степень существенности $R$ равна 1 , справедливо неравенство

$$
C(I) \geqslant n \text {. }
$$

Поскольку реконструирующая функщия существенно зависит только от одного бита из ответов серверов, то этот бит должен быть одним из битов базы данных или его отрицанием. И наоборот, если некоторый бит ответа существенно зависит более чем от одного бита базы данных, то реконструирующая функция не может существенно зависеть от этого бита ответа. Это значит, что если заменить этот бит ответа на любой бит базы данных, то свойство корректности не нарушится и коммуникационная сложность не изменится. Если такую операцию проделать для всех бит ответа, существенно зависящих от более чем одного бита базы данных, то мы получим PIR-протокол с функцией ответа со степенью существенности 1 и коммуникационной сложностью, равной $C(I)$. Но согласно предложению 5 такой PIR-протокол имеет коммуникационную сложность не менее $n$.

Таким образом, первая часть предложения 6 доказана.

Для доказательства второй части предложения 6 достаточно заметить, что у PIR-протокола $I_{g}$ из раздела 4 реконструирующая функция имеет степень существенности 2.

\section{Список литературы}

1. Chor B., Goldreich O., Kushilevitz E., Sudan M., Private information retrieval. J. $A C M$ (1998) 45, №6, 965-982.

2. Гасанов Э. Э., Майлыбаева Г. А., Доступ к базам данных без раскрытия запроса. В сб.: Материалы конференции «Математика и безопасные информачионные технологии». Москва, 2003, c. 393-395. 